\title{
Banach Algebras in Which Every Left Ideal is Countably Generated
}

\author{
NADIA BOUDI
}

\section{INTRODUCTION}

An algebra which is associative or alternative is Noetherian if it satisfies the ascending chain condition on left ideals or equivalently, every left ideal is finitely generated. A well-known result of Sinclair and Tullo [9] states that an associative Noetherian Banach algebra is finite dimensional. This result was extended in [2] to the alternative case. In this paper, we are concerned with associative and alternative Banach algebras in which every left ideal is countably generated. Several papers have appeared dealing with countably generated ideals in some Banach algebras (like in [4], [6]). It should be pointed out that sometimes we have some surprising facts, see for example [7] and [8].

It is clear that we can treat directly the alternative case, since every associative algebra is alternative. But our purpose here is to present the methods rather than the results. And the proof of the alternative case is rather more complicated.

\section{Associative Case}

Throughout, we use 'countable' to mean finite or denumerably infinite. A key result in [9] is the fact that if the closure of a left ideal $I$ of an associative Banach algebra is finitely generated as a left ideal, then $I$ is closed. The next proposition is a generalization of this result.

Given a Banach space $E$ and $X \subseteq E$, we denote by $X^{-}$and $\partial X$, respectively, the closure and the boundary of $X$.

This paper was presented at the "All Ireland Algebra Days 2001" held at Queen's University Belfast during May 16-19, 2001. 
Proposition 1. Let $A$ be a real or complex Banach algebra, and let $I$ be a left ideal of $A$. If the left ideal $I^{-}$is countably generated, then $I$ is closed.

Proof. Let $\left(x_{n}\right)$ be a sequence of elements of $I^{-}$such that $I^{-}=$ $\sum A^{\prime} x_{n}$, where $A^{\prime}$ denotes the unitisation of $A$. For each $n$, let $\left(v_{k}^{n}\right)$ be a sequence of elements of $I$ converging to $x_{n}$. Without loss of generality, we can suppose that $\left\|x_{n}\right\|=\left\|v_{k}^{n}\right\|=1$ and $\left\|x_{n}-v_{k}^{n}\right\|<1$ for each $n, k \in \mathbb{N}$. Set $\Gamma=\bigoplus_{n \in \mathbb{N}^{*}} A_{n}$, where $A_{n}=A^{\prime}$. We define on $\Gamma$ a vector space norm $\|.\|^{\prime}$ with $\left\|\left(a_{n}\right)\right\|^{\prime}=\sum n\left\|a_{n}\right\|$. Let $T$ be the map:

$$
T: \begin{array}{ccc}
\Gamma & \longrightarrow & I^{-} \\
\left(a_{n}\right) & \longmapsto & \sum a_{n} x_{n},
\end{array}
$$

and for each $k$, let $T_{k}$ be the map given by:

$$
\begin{aligned}
& T_{k}: \quad \Gamma \longrightarrow I^{-} \\
& \left(a_{n}\right) \longmapsto \sum_{n=1}^{k} a_{n} v_{n}^{k}
\end{aligned}
$$

Clearly, $T, T_{k}$ are linear and bounded. We claim that the sequence $\left(T_{k}\right)$ converges uniformly to $T$ on $\Gamma$. Indeed, for $\varepsilon>0$, let $0 \neq t$ be in $\mathbb{N}$ with $t \neq 0$ and $1 / t<\epsilon$. Choose $N \in \mathbb{N}$ such that $N>t$, and for each $k>N$ and $n \in\{1, \ldots, t\},\left\|x_{n}-v_{k}^{n}\right\|<1 / t$. Then, for $\left(a_{n}\right)_{n} \in \Gamma$,

$$
\begin{aligned}
\left\|T-T_{k}\left(a_{n}\right)\right\| & \leq\left\|\sum_{n=1}^{t} a_{n}\left(x_{n}-v_{k}^{n}\right)\right\|+\left\|\sum_{t+1}^{k} a_{n}\left(x_{n}-v_{k}^{n}\right)\right\|+\left\|\sum_{k+1}^{\infty} a_{n} x_{n}\right\| \\
& \leq \sum_{n=1}^{t} \frac{1}{t}\left\|a_{n}\right\|+\sum_{t+1}^{\infty}\left\|a_{n}\right\| \\
& \leq \frac{1}{t}\left(\sum_{n=1}^{t}\left\|a_{n}\right\|+\sum_{t+1}^{\infty} t\left\|a_{n}\right\|\right) \\
& \leq \frac{1}{t} \sum_{n=1}^{\infty} n\left\|a_{n}\right\|=1 / t\left\|\left(a_{n}\right)\right\|^{\prime} \\
& <\varepsilon\left\|\left(a_{n}\right)\right\|^{\prime}
\end{aligned}
$$

Denote by $\Lambda$ the completion of the normed vector space $\left(\Gamma,\|\cdot\|^{\prime}\right)$. Of course, $\Lambda=\left\{\left(a_{n}\right) \in \Pi A_{n}: \sum n\left\|a_{n}\right\|<\infty\right\}$. Let $\bar{T}$ (resp. $\left.\bar{T}_{k}\right)$ : $\Lambda \rightarrow I^{-}$be the continuous extension of $T$ (resp. $T_{k}$ ). Then, the sequence of continuous linear operators $\left(\bar{T}_{k}\right)$ converges uniformly to $\bar{T}$, but $\bar{T}$ is surjective and it is well known that the set of surjective continuous linear mappings is open, hence there exists a positive 
integer $k$ such that $\bar{T}_{k}$ is surjective. Now the proof is completed by showing that $\bar{T}_{k}(\Lambda) \subseteq I$. Let $\left(a_{n}\right)_{n}$ be an element of $\Lambda$. Let us consider the sequence $\left(u_{k^{\prime}}\right)_{k^{\prime}}$, where $u_{k^{\prime}}=\left(b_{n}^{k^{\prime}}\right)_{n}$ and

$$
b_{n}^{k^{\prime}}=\left\{\begin{array}{c}
0 \text { if } n>k^{\prime} \\
a_{n} \text { if } n \leq k^{\prime}
\end{array} .\right.
$$

Then, since $\sum n\left\|a_{n}\right\|<\infty, \lim _{k^{\prime}} u_{k^{\prime}}=\left(a_{n}\right)_{n}$. Clearly, $\bar{T}_{k}\left(\left(a_{n}\right)_{n}\right)=$ $\lim _{k^{\prime}} T_{k}\left(u_{k^{\prime}}\right)$. For each $k^{\prime}>k, T_{k}\left(u_{k^{\prime}}\right)=\sum_{n=1}^{k} b_{n}^{k^{\prime}} v_{n}^{k}=\sum_{n=1}^{k} a_{n} v_{n}^{k}$. Hence, $\left(T_{k}\left(u_{k^{\prime}}\right)\right)_{k^{\prime}}$ stabilizes. Therefore, for each $k \in \mathbb{N}, \bar{T}_{k}\left(\left(a_{n}\right)_{n}\right)=$ $\sum_{n=1}^{k} a_{n} v_{n}^{k} \in I$, and the proof is complete.

The following result was shown by Sidney; see [1, p. 77] for a short simple proof.

Theorem 2. Let $A$ be a Banach algebra. If every left ideal of $A$ is closed, then $A$ is finite dimensional.

Combining Proposition 1 with the above theorem, we obtain the main result of this section.

Theorem 3. Let $A$ be a Banach algebra. If every closed left ideal is countably generated, then $A$ is finite dimensional.

\section{Alternative Case}

A nonassociative algebra $A$ over a field $K$ of characteristic zero is said to be an alternative algebra if it satisfies:

$$
x^{2} y=x(x y) ; \quad y x^{2}=(y x) x
$$

for all $x, y \in A$. Let $A$ be an alternative algebra. Then $A$ is called semiprime (respectively, prime) if for every ideal $I$ of $A$ (resp., for every two of its ideals $I$ and $J$ ) it follows from $I^{2}=(0)$ (resp. $I J=(0)$ ) that $I=(0)$ (resp., that either $I=(0)$ or $J=(0)$ ). Let $X$ be a subset of $A$. The annihilator of $X$ is defined by $\operatorname{ann}(X)=$ $\{a \in A: X a=a X=0\}$. If the center $Z(A)$ of $A$ is nonzero and does not contain zero divisors of the algebra $A, A$ is said to be a Cayley Dickson ring if moreover the ring of quotients $\left(Z(A)^{*}\right)^{-1}$ is a Cayley Dickson algebra over the field of quotients of the center $Z(A)$ (where $Z(A)^{*}=Z(A) \backslash\{0\}$ ). One can prove that in $A$, there exists a smallest ideal $B(A)$ such that $A / B(A)$ does not contain nonzero trivial ideals [10]; $B(A)$ is called the Baer radical of $A$. 
A real or complex nonassociative algebra $A$ is said to be normed (respectively, Banach) algebra if the underlying vector space of $A$ is endowed with a norm (respectively, complete norm) $\|\cdot\|$ satisfying $\|a b\| \leq\|a\|\|b\|$ for all $a, b \in A$. Any alternative algebra $A$ can be imbedded in a unital alternative algebra $A^{\prime}, A^{\prime}=K+A$. For basic results on alternative algebras, the reader is referred to [10]. In particular, recall that every prime alternative algebra $A$ that is not associative is a Cayley Dickson ring. Also, recall that if $A$ is an alternative algebra, then for any two of its ideals $I$ and $J$, the product $I J$ is also an ideal of the algebra $A$. Now, let $A$ be a nonassociative Banach algebra and let $B L(A)$ denote the algebra of all bounded linear operators on $A$. For $a$ in $A, L_{a}$ will mean the operator of left multiplication by $a$ on $A$; clearly, $L_{a}$ is bounded. We will denote by $L(A)$ the left multiplication algebra of $A$, namely, the subalgebra of $B L(A)$ generated by the identity operator $i d_{A}$ and the set $\left\{L_{a}: a \in A\right\}$.

Let $A$ be a nonassociative algebra and let $I$ be a left ideal of $A$. If $I$ is generated by $\left\{x_{i}\right\}$, then $I=\sum L(A) x_{i}$. Note that if $A$ is endowed with a complete norm, then $L(A)$ need not be closed. Hence, the technique of the proof of Proposition 1 gives a restricted version of this proposition in the nonassociative context, but which is still enough for our purposes.

Proposition 4. Let $A$ be a nonassociative real or complex Banach algebra, and let $I$ be a left ideal of $A$. If $L(A)^{-} I \subseteq I$ and $I^{-}$is countably generated as a left ideal in $A$, then $I$ is closed.

The next lemma is an immediate consequence of the above proposition.

Lemma 5. Let $A$ be a nonassociative real or complex Banach algebra, and let $y$ be an element of the nucleus $N(A)$ of $A$. If $\left(A^{\prime} y\right)^{-}$ is countably generated as a left ideal in $A$, then $A^{\prime} y$ is closed.

Let $A$ be an algebra. We will denote by $i d\left(a_{1}, \ldots, a_{n}\right)$ the ideal generated by $a_{1}, \ldots, a_{n} \in A$.

Lemma 6. Let $A$ be an alternative semiprime complex unital Banach algebra in which every left ideal is countably generated. Then the center $Z(A)$ is semisimple and finite dimensional.

Proof. Let us first prove that $Z(A)$ has finite spectrum. Let $x$ be in $Z(A)$ and assume that $\operatorname{sp}(x, Z(A))$ is infinite. Then, $\partial \operatorname{sp}(x, Z(A))$ is infinite. Consider the set 


$$
I=\left\{z \in A \mid \exists \lambda_{1}, \ldots, \lambda_{n} \in \partial \operatorname{spx}, z\left(x-\lambda_{1}\right) \ldots\left(x-\lambda_{n}\right)=0\right\} .
$$

Clearly, $I$ is an ideal of $A$ and $L(A)^{-} I \subseteq I$. Hence, $I$ is closed (Proposition 4). By assumption, we can find a sequence $\left(z_{n}\right)_{n} \subseteq I$ such that $I=\sum_{n} L(A) z_{n}$. For each $n$, there exist $\lambda_{1}^{n}, \ldots, \lambda_{r_{n}}^{n} \in \partial \operatorname{spx}$ $\left(r_{n} \in \mathbb{N}\right)$ such that

$$
z_{n}\left(x-\lambda_{1}^{n}\right) \cdots\left(x-\lambda_{r_{n}}^{n}\right)=0 .
$$

If $\partial \operatorname{spx}$ is not countable, then we can pick $\lambda \in \partial s p x$ so that $\lambda \notin$ $\left\{\lambda_{i}^{n}: i=1, \ldots, r_{n}, n \in \mathbb{N}\right\}$. Define

$$
\begin{aligned}
T: A & \longrightarrow A \\
y & \longmapsto y x .
\end{aligned}
$$

We check easily that $s p T=s p(x, Z(A))=s p(x, A)$. Thus, $\lambda_{n} \in$ $\partial \operatorname{spT}$. Since $A(x-\lambda)$ is closed (Lemma 5 ), we can apply [1, Lemma 3 on p. 75] to $\lambda$ to get $z \in A$ with $z(x-\lambda)=0$. Hence, $z \in I$ and $z=S_{1} z_{1}+\ldots+S_{n} z_{n}$ for some $S_{1}, \ldots, S_{n} \in L(A)$. Therefore,

$$
z \prod_{\substack{i=1, \ldots, n \\ j=1, \ldots, r_{n}}}\left(x-\lambda_{j}^{i}\right)=0
$$

and thus $\prod_{i, j}\left(\lambda-\lambda_{j}^{i}\right)=0$, which is impossible. Consequently, $\partial s p x$ is countable. Set $\partial \operatorname{spx}=\left\{\lambda_{n} \mid n \in \mathbb{N}\right\}$. Then, we can write $I=\bigcup_{n \in \mathbb{N}} I_{n}$, where $I_{n}=\left\{z \in A \mid z\left(x-\lambda_{1}\right) \cdots\left(x-\lambda_{n}\right)=0\right\}$. Using Baire's Theorem and Proposition 4, we deduce that $I=\bigcup_{n=1}^{N} I_{n}=I_{N}$ for some $N \in \mathbb{N}$, and we proceed as above to prove that $\partial$ spx is finite. Now we prove that $Z(A)$ is semisimple. Let $x$ be in $\operatorname{Rad} Z(A)$. Then, $x$ is quasi-nilpotent. Set $J=\left\{a \in A \mid \exists n \in \mathbb{N}, a x^{n}=0\right\} ; J$ is an ideal of $A, L(A)^{-} J \subseteq J$ and hence, $J$ is closed (Proposition 4). Furthermore, $J=\bigcup_{n \in \mathbb{N}} J_{n}$ where $J_{n}=\left\{a \in A: a x^{n}=0\right\}$. Again by Baire's Theorem and Proposition 4, there exists $n \in \mathbb{N}$ such that $J=\bigcup_{k=1}^{n} J_{k}=J_{n}$. Suppose that $J_{n} \neq A$. Consider

$$
\begin{array}{ccc}
T: \quad A / J_{n} & \longrightarrow & A / J_{n} \\
a+J_{n} & \longmapsto & a x+J_{n} .
\end{array}
$$

Clearly, $T$ is quasi-nilpotent. Applying again [1, Lemma 3 on p. 75], we get $a \in A$ such that $a \notin J_{n}$ and $a x \in J_{n}$. Hence, $a \in J_{n+1}=$ $J_{n}$ which is impossible. Thus, $J_{n}=A$ and $x$ is nilpotent. Since $x \in Z(A), i d(x)$ is nilpotent and hence, by the semiprimeness of $A$, 
$x=0$. Finally, the lemma follows from the well-known result of Kaplansky [5].

Lemma 7. Let $A$ be a complex alternative prime Banach algebra in which every left ideal is countably generated. Suppose that $A$ is not associative; then, $A=\mathbb{O}_{\mathbb{C}}$ (the Cayley Dickson algebra over $\mathbb{C}$ ).

Proof. Since $A$ is prime, $Z(A)$ does not contain non-zero zero divisors. Furthermore, by Lemma $6, Z(A)$ is finite dimensional and semisimple. Hence, by Wedderburn's theorem for semisimple finite dimensional associative complex algebras, it is isomorphic to the complex field. Now, the lemma is a consequence of [10, p. 194].

We are now in a position to prove the main theorem of this section.

Theorem 8. Let $A$ be an alternative complex Banach algebra. If every left ideal is countably generated, then $A$ is finite dimensional.

Proof. If $A$ is not unital, then $A^{\prime}$ is a complex alternative Banach algebra in which every left ideal is countably generated. Hence, we can assume without loss of generality that $A$ is unital.

Let us first examine the semiprime case. Then, every associative ideal $I$ of $A$ is contained in the nucleus $N(A)$ of $A(N(I)=I \cap N(A)$ [10, p. 177]. Let $U$ be the largest associative ideal of $A$. Clearly, $U$ is closed, $A / U$ is purely alternative and semiprime (for the second fact, one can use for example the semiprimeness of $A$ and the well known property: $(A, A, A) U=0[10$, p. 136, Lemma 1], also, using the same identities, one can deduce the third fact). Now, set $\bar{A}=A / U$. Then, $\bar{A}$ is a purely alternative semiprime Banach algebra in which every left ideal is countably generated, hence for every nonzero ideal of $\bar{A}, I \cap Z(\bar{A}) \neq 0[10]$. Pick a prime ideal $P_{1}$ of $\bar{A}$. Then, since $Z(\bar{A})$ is finite dimensional (Lemma 7 ) and $\bigcap_{P \text { prime }} P=0[10]$, there exists a prime ideal $P_{2}$ of $\bar{A}$ such that $P_{2} \cap P_{1} \cap Z(\bar{A}) \neq P_{1} \cap Z(\bar{A})$. Hence, we can proceed analogously to prove that $\bigcap_{i=1}^{n} P_{i} \cap Z(\bar{A})=0$ for some prime ideals $P_{3}, \ldots, P_{n}$. And so, $\bigcap_{i=1}^{n} P_{i}=0$. Without loss of generality we can assume $P_{i} \nsubseteq P_{j}$ if $i \neq j$. Then each $P_{i}$ is closed $\left(P_{i}=\operatorname{ann}\left(\bigcap_{j \neq i} P_{j}\right)\right)$, hence $\bar{A} / P_{i}$ is finite dimensional (Lemma 7 and Theorem 3 ), and therefore, $\bar{A}$ is finite dimensional. Next we show that $A$ is finite dimensional. Let $I$ be a left ideal of $U$. Let $\left(x_{n}\right)$ be 
a sequence of $I$ such that $A^{\prime} I A^{\prime}=\sum A^{\prime} x_{n}$. Then, $I=\sum U x_{n}+F$ where $F$ is a countably dimensional space. Hence, every left ideal of $U$ is countably generated and Theorem 3 yields that $U$ is finite dimensional.

Now, for the general case, let us consider the Baer chain of ideals $\left[10\right.$, p. 161], $B_{1}(A) \subseteq B_{2}(A) \ldots \subseteq B_{n}(A) \ldots$ and let $B^{\prime}(A)=$ $\bigcup B_{i}(A)$. Since $L(A)^{-} B_{1}(A) \subseteq B_{1}(A), B_{1}(A)$ is closed (Propo$i \in \mathbb{N}$

sition 4). Now, by considering $A / B_{i-1}(A)$, we deduce that $B_{i}(A)$ is closed for each $i$. And so, Baire's Theorem and Proposition 4 show that $B^{\prime}(A)=\bigcup_{i=1}^{n} B_{i}(A)$ for some $n$, which implies that $B^{\prime}(A)=$ $B_{n}(A)$. And consequently, the Baer radical of $A$ is $B_{n}(A)$. Since $B_{1}(A)$ is a countably generated left ideal, we can choose a sequence $\left(a_{n}\right)_{n \in \mathbb{N}}$ of elements of $A$ such that $i d\left(a_{n}\right)$ is trivial and $B_{1}(A)=$ $\bigcup i d\left(a_{1}, \ldots a_{i}\right)$. Hence, by applying Baire's Theorem and Proposition 4 , we infer that $B_{1}(A)=\bigcup_{i=1}^{N}\left(i d\left(a_{1}, \ldots, a_{i}\right)\right)^{-}=\left(i d\left(a_{1}, \ldots, a_{N}\right)\right)^{-}$ for some $N \in \mathbb{N}$. Now, we check easily that $B_{1}(A)$ is nilpotent. Set $\bar{A}=A / B_{n-1}(A)$. Then, $\bar{A}$ is a complex alternative Banach algebra in which every left ideal is countably generated. Therefore, $B_{1}(\bar{A})=B_{n}(A) / B_{n-1}(A)$ is nilpotent. Choose $k \in \mathbb{N}$ such that $\left(B_{1}(\bar{A})\right)^{k}=0$. Then, $\left(\left(B_{1}(\bar{A})\right)^{k-1}\right)^{-}$is a countably generated module over $L\left(\bar{A} / B_{1}(\bar{A})\right)$. But $\bar{A} / B_{1}(\bar{A}) \simeq A / B_{n}(A)$ which is finite dimensional, hence $L\left(\bar{A} / B_{1}(\bar{A})\right)$ is countably dimensional and so, $\left(\left(B_{1}(\bar{A})\right)^{k-1}\right)^{-}$is countably dimensional. Thus, by Baire's Theorem, $\left(B_{1}(\bar{A})\right)^{k-1}$ is finite-dimensional. Consider $\left(B_{1}(\bar{A})\right)^{k-2} /\left(B_{1}(\bar{A})\right)^{k-1}$ and $\bar{A} /\left(B_{1}(\bar{A})\right)^{k-1}$, as above, we show that $\left(B_{1}(\bar{A})\right)^{k-2}$ is finite dimensional. We continue in this fashion to obtain that $B_{1}(\bar{A})$ is finite dimensional, hence $\bar{A}$ is finite dimensional. A recursive argument allows us to show that $A$ is finite dimensional, which completes the proof.

Corollary 9. Let $A$ be a real alternative Banach algebra in which every left ideal is countably generated. Then $A$ is finite dimensional.

Proof. Consider $A_{\mathbb{C}}=A+i A$, the complexification of $A$. Then, by a straightforward argument, we show that every left ideal is countably generated in $A_{\mathbb{C}}$. Also, recall that $A_{\mathbb{C}}$ is endowed with a complete norm [3]. Now, by applying Theorem 8 we deduce that $A_{\mathbb{C}}$ and hence $A$ is finite dimensional. 


\section{ACKNOWLEDGEMENTS}

I would like to thank Miguel Gomez Lozano for several discussions during the "All Ireland Algebra Days 2001" in Belfast which enabled me to deduce the alternative semiprime case from the prime one. I also wish to express my thanks to El Amin Kaidi and Martin Mathieu for the support during the "All Ireland Algebra Days 2001". And I thank the referee for his valuable comments.

\section{REFERENCES}

[1] B. Aupetit, Propriétés spectrales des algèbres de Banach, Lecture Notes in Math. Vol. 735, Springer-Verlag, Berlin New York, 1979.

[2] M. Benslimane and N. Boudi, Alternative Noetherian Banach algebras, Extracta Math. 12 (1997), 41-46.

[3] F. F. Bonsall and J. Duncan, Complete normed algebras, Springer-Verlag, Berlin Heidelberg, New York, 1973.

[4] L. Gillman, Countably generated ideals in rings of continuous functions, Proc. Amer. Math. Soc. 11 (1960), 660-666.

[5] I. Kaplansky, Ring isomorphisms of Banach algebras, Canad. J. Math. 6 (1954), 374-381.

[6] A. LeDonne, On countably generated z-ideals of $C(X)$ for first countable spaces, Proc. Amer. Math. Soc. 82 (1981) 280-282.

[7] R. Mortini, The Forelli problem concerning ideals in the disk algebra $A(D)$. Preprint.

[8] R. Mortini, Countably generated prime ideals in algebras of analytic functions. Complex Variables, Theory Appl. 14, No. 1-4 (1990), 215-222.

[9] A. M. Sinclair and A. W. Tullo, Noetherian Banach algebras are finite dimensional, Math. Ann. 211 (1974), 151-153.

[10] A. Zhevlakov, A. M. Slin'ko, I. P. Shestakov and A. I. Shirshov, Rings that are nearly associative, Pure Appl. Math. Vol. 104, Academic Press, New York London, 1982.

Nadia Boudi,

Département de Mathématiques,

Faculté des Sciences,

B. P. 2121, Tétouan, Maroc

nadia_boudi@yahoo.ca

Received on 2 October 2001 and in revised form on 30 May 2002. 